\title{
基于 $\mathrm{PA}_{2}\left(\mathrm{CsPbBr}_{3}\right)_{n-1} \mathrm{PbBr}_{4}$ 二维层状钙钛矿的 电致发光二极管
}

孟妍 ${ }^{1}$, 牛连斌 ${ }^{2 *}$, 许龙 ${ }^{1}$, 林春燕 ${ }^{3}$, 熊自阳 ${ }^{1}$, 熊祖洪 ${ }^{1}$, 陈平 $1^{*}$

1. 西南大学物理科学与技术学院, 发光与实时分析教育部重点实验室, 重庆 400715;

2. 重庆师范大学物理与电子工程学院, 重庆市光电功能材料重点实验室, 重庆 401331;

3. 西南大学材料与能源学部, 重庆市洁净能源与先进材料研究院, 重庆 400715

* 联系人, E-mail: niulb03@126.com; chenping206@126.com

2017-06-28 收稿, 2017-07-20 修回, 2017-07-20 接受, 2017-09-01 网络版发表

国家自然科学基金(11504300，11374242)、重庆市基础与前沿研究计划(cstc2015jcyjA50002，CSTC2015jcyjA70001，CSTC2015jcyjBX0032) 和中央高校基本业务费专项(XDJK2017D140, XDJK2017C061)资助

摘要进一步提高全无机卤铅钙钛矿材料 $\mathrm{CsPbBr}_{3}$ 的发光效率, 对制备高效率、高稳定性的电致发光二极管 (PeLED)具有重要意义. 制备纳米级的钙钛矿量子点, 一方面有助于提高激子的束缚能和钻钛矿晶体的苂光效率, 另一方面也有利于形成连续、致密的二维层状钙钛矿薄膜. 本文采用“原位生长”的策略, 将一种具有长链结构的 丙基渲化胺 $\left(\mathrm{CH}_{3} \mathrm{CH}_{2} \mathrm{CH}_{2} \mathrm{NH}_{3} \mathrm{Br}, \mathrm{PABr}\right)$ 作为添加剂, 与 $\mathrm{CsPbBr}$ 的前驱体溶液进行共混, 得到 $\mathrm{PA}_{2}\left(\mathrm{CsPbBr}_{3}\right)_{n-1} \mathrm{PbBr}_{4}$ 钙钛矿量子点. 形成的二维层状钻钛矿薄膜均匀致密, 在光致发光条件下, 呈现出明亮的蓝绿光发射(发光峰位于 $506 \mathrm{~nm})$. 在电致发光方面, 基于 $\mathrm{PA}_{2}\left(\mathrm{CsPbBr}_{3}\right)_{n-1} \mathrm{PbBr}_{4}$ 的PeLED启亮电压为 $4.2 \mathrm{~V}$, 最大亮度为 $2370 \mathrm{~cd} / \mathrm{m}^{2}$, 最高 电流效率为 $1.06 \mathrm{~cd} / \mathrm{A}$, 最高 $\mathrm{EQE}$ 为 $\sim 0.57 \%$. 相较于传统方法, 本工作在制作工艺、成膜质量以及PeLED的发光效 率有了显著的提升, 为将来进一步探索低成本、高效率的蓝光PeLEDs提供了一种可行的思路.

关键词 $\mathrm{PA}_{2}\left(\mathrm{CsPbr}_{3}\right)_{n-1} \mathrm{PbBr}_{4}$, 二维层状, 钻钛矿, 发光二极管

近年来，基于卤铅钲钛矿材料的电致发光二极 管(PeLED)迅速成为全世界科学家的研究热点 ${ }^{[1 ~ 9]}$, PeLED的效率正在不断攀升. 目前, 以甲基胺与卤化 铅合成的“有机-无机杂化钙钛矿” $\left(\mathrm{CH}_{3} \mathrm{NH}_{3} \mathrm{PbBr}_{3}\right)$, 其 PeLED的外量子效率(EQE) 已经高达 $8.53 \%{ }^{[7]}$, 与传 统的有机发光二极管 (OLED)的水平相当. 尽管有机无机杂化钙钛矿在效率方面已经取得了巨大的进步, 然而稳定性差却成为制约其进一步发展的瓶颈问题. 首先, 有机-无机杂化钙钛矿对工作环境的水、氧分 子十分敏感: 在潮湿的空气中, 材料很容易发生分 解 ${ }^{[10]}$. 其次, 有机-无机杂化钻钛矿的热稳定性也较
差, PeLED工作时产生的焦耳热会使器件性能快速地 发生衰减 ${ }^{[9]}$. 相比之下, 全无机的卤铅钙钛矿材料 $\mathrm{CsPbBr}_{3}$ 具有更良好的稳定性 ${ }^{[11]}$. 因此, 进一步提高 $\mathrm{CsPbBr}_{3}$ 的发光效率, 有望制备出效率高并且稳定性 良好的PeLED, 进而帮助卤铅钙钛矿材料在平板照 明和显示领域实现产业化.

对于三维(3D)的钙钛矿晶体 (晶体尺寸在 0.5 至数 个微米之间), 由于电子-空穴对的距离较远, 激子的 束缚能很小 (通常小于 $100 \mathrm{meV}$ ), 因此激子往往以非 辐射退激的方式退激, 从而降低钙钛矿晶体的苂光效 率 $^{[12]}$. 此外, $3 \mathrm{D}$ 钲钛矿晶体还不利于形成连续、致密

引用格式: 孟妍, 牛连斌, 许龙, 等. 基于 $\mathrm{PA}_{2}\left(\mathrm{CsPbBr}_{3}\right)_{n-1} \mathrm{PbBr}_{4}$ 二维层状钻针矿的电致发光二极管. 科学通报, 2018, 63: 53-60 Meng Y, Niu L B, Xu L, et al. Light-emitting diodes based on two-dimensional $\mathrm{PA}_{2}\left(\mathrm{CsPbBr}_{3}\right)_{n-1} \mathrm{PbBr}_{4}$ layered perovskites (in Chinese). Chin Sci Bull, 2018, 63: 53-60, doi: 10.1360/N972017-00712 
的钙钛矿薄膜, 导致器件中存在显著的漏电流 ${ }^{[13]}$. 有 研究工作表明, 减小钙钛矿晶体的尺寸(例如, 制备纳 米级的钙钛矿量子点), 一方面有助于提高激子的束 缚能和钙钛矿晶体的苂光效率, 另一方面也有利于形 成连续、致密的二维层状钙钛矿薄膜 ${ }^{[3,4]}$. 在先前的工 作中, 人们主要通过溶液法来合成 $\mathrm{CsPbr}_{3}$ 钙钛矿量 子点 ${ }^{[14]}$. 然而, 该方法主要面临以下 3 个方面的问题: (1) 合成方法复杂: 合成过程中需要反复清洗和离心, 才能得到最终产物 ${ }^{[14]}$; (2) 长链的绝缘配体(例如, 油 氨和油酸)会包覆在钻钛矿量子点的表面, 从而影响 $\mathrm{CsPbBr}_{3}$ 钙钛矿的电学传输性能 ${ }^{[4]}$; (3) 钲钛矿量子点的 溶解度十分有限 (仅为 $0.5 \mathrm{mg} / \mathrm{mL}$ ) ${ }^{[14]}$, 所以难以通过溶 液加工得到理想的钙钛矿薄膜. 因此, 人们迫切地需 要一种合成简单、不影响钙钛矿的电学传输性能, 并且 可用于溶液加工的 $\mathrm{CsPbBr}_{3}$ 钻钛矿量子点合成方法.

本工作采用一种“原位生长”的策略 ${ }^{[3,4]}$ 得到钙钛 矿量子点. 将一种具有长链结构的丙基溴化胺 $\left(\mathrm{CH}_{3} \mathrm{CH}_{2} \mathrm{CH}_{2} \mathrm{NH}_{3} \mathrm{Br}, \mathrm{PABr}\right)$ 作为添加剂, 按 $0.71: 1$ 摩 尔比与 $\mathrm{CsPbBr}_{3}$ 的前驱体溶液进行共混, 避免引人长 链有机分子导致的材料电阻率上升. 最后采用一步 旋涂的方法成功制备了 $\mathrm{PA}_{2}\left(\mathrm{CsPbBr}_{3}\right)_{n-1} \mathrm{PbBr}_{4}$ 的二维 层状钙钛矿薄膜. XRD结果表明, 一旦PA离子取代 $\mathrm{CsPbBr}_{3}$ 钙钛矿中部分 $\mathrm{Cs}$ 离子, 其长链结构就可以有 效地阻止 $\mathrm{CsPbr}_{3}$ 钙钛矿单元的进一步自组装生长, 从而得到纳米级的 $\mathrm{PA}_{2}\left(\mathrm{CsPbBr}_{3}\right)_{n-1} \mathrm{PbBr}_{4}$ 钙钛矿量子 点. $\mathrm{SEM}$ 表征结果显示, 由 $\mathrm{PA}_{2}\left(\mathrm{CsPbr}_{3}\right)_{n-1} \mathrm{PbBr}_{4}$ 钙钛 矿量子点组成的二维层状钙钛矿薄膜, 均匀致密, 成 膜质量优异. 在 365 nm紫外灯照射下, $\mathrm{PA}_{2}(\mathrm{CsPb}-$ $\left.\mathrm{Br}_{3}\right)_{n-1} \mathrm{PbBr}_{4}$ 钻钛矿薄膜呈现明亮的蓝绿光发射 (发光 峰位于 $506 \mathrm{~nm})$. 在电致发光方面, 基于 $\mathrm{PA}_{2}(\mathrm{CsPb}-$ $\left.\mathrm{Br}_{3}\right)_{n-1} \mathrm{PbBr}_{4}$ 的PeLED同样给出 $506 \mathrm{~nm}$ 的蓝绿光发射, 启亮电压为 $\sim 4.2 \mathrm{~V}$, 最大亮度为 $2370 \mathrm{~cd} / \mathrm{m}^{2}$, 最高电 流效率为 $1.06 \mathrm{~cd} / \mathrm{A}$, 最高 $\mathrm{EQE}$ 为 $\sim .57 \%$. 该PeLED 的结果要明显优于传统通过卤化物取代得到的蓝光 PeLED 的结果 ${ }^{[8]}$. 本工作制备了 $\mathrm{PA}_{2}\left(\mathrm{CsPbBr}_{3}\right)_{n-1^{-}}$ $\mathrm{PbBr}_{4}$ 的二维层状钙钛矿薄膜, 并研究了其光致发光 和电致发光特性. 制作工艺简单可靠, 也为将来进一 步探索低成本、高效率的蓝光PeLEDs提供了一种可 行的思路.

\section{1 实验与测量}

首先在手套箱中 (水、氧含量 $<0.1 \mathrm{ppm}$ ) 配制
$\mathrm{CsPbBr}_{3}$ 的前驱体溶液. 将 $\mathrm{CsBr}$ (Alfa Aesar, 纯度 $>99.999 \%$ ) 和 $\mathrm{PbBr}_{2}$ (西安宝莱特光电科技有限公司, 纯度 $>99.9 \%$ ) 以 1:1摩尔比混合溶解在二甲基亚砜 (DMSO, 上海阿拉丁生化科技股份有限公司, 色谱 级, 纯度 $>99.8 \%$ ) 溶剂中, 室温搅拌超过 $12 \mathrm{~h}$ 后得到 $10 \mathrm{wt} \%$ 的 $\mathrm{CsPbBr}_{3}$ 溶液. $\mathrm{PABr}$ (西安宝莱特光电科技 有限公司, 纯度 $>99.9 \%$ ) 的DMSO溶液同样也在手套 箱中配制而成, 浓度为 $100 \mathrm{mg} / \mathrm{mL}$. 之后, 将 $\mathrm{PABr}$ 溶 液与 $\mathrm{CsPbBr}_{3}$ 前驱体溶液进行混合, 摩尔比为 $\mathrm{PABr}$ ： $\mathrm{CsPbBr}_{3}=0.71: 1^{[3,4]}$. 当 $\mathrm{PABr}$ 掺杂浓度过低时, $\mathrm{PA}$ 离 子对 $\mathrm{Cs}$ 离子的取代程度有限, 无法有效地形成 $\mathrm{PA}_{2}\left(\mathrm{CsPbrr}_{3}\right)_{n-1} \mathrm{PbBr}_{4}$ 量子点. 当 $\mathrm{PABr}$ 掺杂浓度过高 时, $\mathrm{PABr}$ 的长链又会导致器件本身的电阻明显上升, 不利于载流子的注人与传输. 因此, $\mathrm{PABr}$ 与 $\mathrm{CsPbBr}_{3}$ 掺杂浓度须保持在一个合适的水平. 室温搅拌 $3 \mathrm{~h}$ 后 得到最终的 $\mathrm{PA}_{2}\left(\mathrm{CsPbBr}_{3}\right)_{n-1} \mathrm{PbBr}_{4}$ 混合溶液.

采用一步旋涂工艺和热阻蒸发工艺制备PeLED. PeLED的结构为 ITO (深圳湘城华南科技有限公 司)/PEDOT:PSS(AI4083, CLEVIOS)/ $/ \mathrm{PA}_{2}\left(\mathrm{CsPbBr}_{3}\right)_{n-1}$ $\mathrm{PbBr}_{4} / \mathrm{TPBi}(1,3,5$-Tris(1-phenyl-1H-benzimidazol-2-yl) benzene, 江苏方晟光电科技有限公司, 纯度 $>99 \%$ )/ $\mathrm{Cs}_{2} \mathrm{CO}_{3}$ (Alfa Aesar, 纯度 $>99.9 \%$ )/Al. 其中, ITO为阳 极, PEDOT: PSS 作为空穴注人层, $\mathrm{PA}_{2}\left(\mathrm{CsPbBr}_{3}\right)_{n-1}$ $\mathrm{PbBr}_{4}$ 作为发光层, $\mathrm{TPBi}$ 作为电子传输层, $\mathrm{Cs}_{2} \mathrm{CO}_{3} / \mathrm{Al}$ 作为阴极. 器件结构如图1所示. 整个PeLED的制备过 程与本课题组 ${ }^{[13,15]}$ 先前报道的工作一致. PeLEDs的有 效面积为 $\sim \mathrm{mm}^{2}$, 制备完毕后在手套箱中进行封装.

$\mathrm{PA}_{2}\left(\mathrm{CsPbBr}_{3}\right)_{n-1} \mathrm{PbBr}_{4}$ 钻钛矿薄膜的紫外吸收谱 由岛津紫外分光光度计(Shimadzu UV-2600)测得, 苂 光光谱(PL谱)由Hitachi F-2500苂光分光光度计测得, 钲钛矿薄膜的表面形貌由扫描电子显微镜 SEM

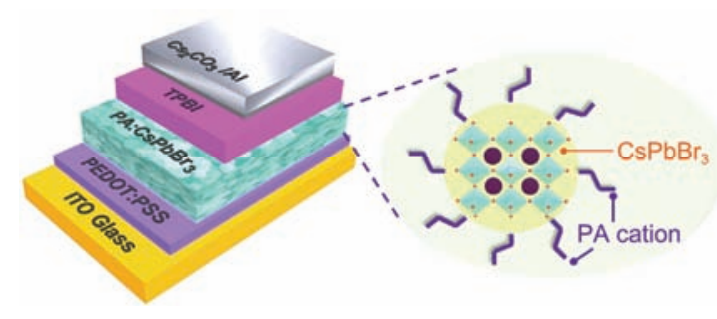

图 1 (网络版彩色)基于 $\mathrm{PA}_{2}\left(\mathrm{CsPbBr}_{3}\right)_{n-1} \mathrm{PbBr}_{4}$ 二维层状钻针矿 $\mathrm{PeLED}$ 的器件结构和 $\mathrm{PA}_{2}\left(\mathrm{CsPBr}_{3}\right)_{n-1} \mathrm{PbBr}_{4}$ 钙针矿量子点结构示意图

Figure 1 (Color online) The scheme of PeLED structure with 2D $\mathrm{PA}_{2}\left(\mathrm{CsPbBr}_{3}\right)_{n-1} \mathrm{PbBr}_{4}$ perovskites emitting layer, and that of $\mathrm{PA}_{2}\left(\mathrm{CsPbBr}_{3}\right)_{n-1} \mathrm{PbBr}_{4}$ quantum dots 
(JEOL, JSM-7100 F) 观察, 钻钛矿薄膜结晶状况由 X射 线粉末衍射仪(岛津, XRD-7000)得到. PeLED的电流亮度-电压 $(I-L-V)$ 特性曲线由 Keithley2400电源和经 过校正的硅光电探头 (北京师范大学光电仪器厂, ST-86LA)共同测得. PeLED的电致发光光谱(EL谱)由 PR670光谱光度计采集. 上述所有测量, 都是在室温大 气环境中进行.

\section{2 结果与讨论}

\section{1 $\mathrm{PA}_{2}\left(\mathrm{CsPbBr}_{3}\right)_{n-1} \mathrm{PbBr}_{4}$ 钻钛矿薄膜的SEM和 XRD表征}

通过“原位生长法”得到钙钛矿量子点的机理如 下: 当 $\mathrm{PABr}$ 与 $\mathrm{CsPbBr}_{3}$ 共混时, $\mathrm{PA}$ 离子可以取代 $\mathrm{CsPbBr}_{3}$ 钙钛矿中部分 $\mathrm{Cs}$ 离子. 此时, $\mathrm{PA}$ 离子的长链 结构无法允许八面体的 $\mathrm{PbBr}_{4}$ 再继续进行自组装. 于 是, 原先三维 $\mathrm{CsPbr}_{3}$ 钙钛矿晶体的生长受到了极大 抑制, 从而形成了 $\mathrm{PA}_{2}\left(\mathrm{CsPbr}_{3}\right)_{n-1} \mathrm{PbBr}_{4}$ 钻钛矿量子 点, 其结构如图 1所示. 利用简单的一步旋涂工艺, $\mathrm{PA}_{2}\left(\mathrm{CsPBBr}_{3}\right)_{n-1} \mathrm{PbBr}_{4}$ 钙钛矿量子点就可以形成二维 层状钙钛矿薄膜(即Ruddlesden-Popper结构 $)^{[16]}$.

首先, 对 $\mathrm{PA}_{2}\left(\mathrm{CsPbBr}_{3}\right)_{n-1} \mathrm{PbBr}_{4}$ 钻钛矿薄膜进行 $\mathrm{SEM}$ 表征, 如图2(a)所示. 可以看到, $\mathrm{PA}_{2}\left(\mathrm{CsPbBr}_{3}\right)_{n-1^{-}}$ $\mathrm{PbBr}_{4}$ 钙钛矿量子点的晶体尺寸很小 (粒径 $<100 \mathrm{~nm}$ ), 均匀致密地形成了一层二维薄膜(nanoplatelet). 并且 晶粒与晶粒之间几乎没有形成孔洞, 薄膜覆盖度几 乎达到 $100 \%$. 因此, 在 $\mathrm{PA}_{2}\left(\mathrm{CsPbBr}_{3}\right)_{n-1} \mathrm{PbBr}_{4}$ 二维层 状钙钛矿薄中, 电子-空穴复合效率和激子辐射退机 速率将得到显著的提升.

图 2(b) 展示了 $\theta-2 \theta$ 的 XRD扫描结果. 这里, $\mathrm{PA}_{2}\left(\mathrm{CsPbBr}_{3}\right)_{n-1} \mathrm{PbBr}_{4}$ 钙钛矿薄膜同样也是沉积在 ITO/PEDOT: PSS基底上. 可以看到, 在 $5^{\circ}, 15.3^{\circ}$ 和 $31.2^{\circ}$ 这 3 个角度上出现了明显的衍射峰. 而对于 $\mathrm{CsPbr}_{3}$ 钻钛矿晶体 (bulk Perovskites), 其XRD衍射 峰出现在 $14^{\circ}$ 和 $29^{\circ}$ 两个角度, 分别对应于 (100)面和 (200)面 ${ }^{[17,18]}$. 这说明PABr引入 $\mathrm{CsPbBr}_{3}$ 后, 钻钛矿的 晶体结构发生了变化. 根据Weidman等人 ${ }^{[19]}$ 对二维 钙钛矿材料的理论模拟结果, 小角度 $5^{\circ}$ 的衍射峰归 属于 $\mathrm{PA}_{2} \mathrm{PbBr}_{4}$ 钙钛矿量子点 (即 $\mathrm{PA}_{2}\left(\mathrm{CsPbBr}_{3}\right)_{n-1^{-}}$ $\left.\mathrm{PbBr}_{4}, n=1\right)$, 而在 $15.3^{\circ}$ 和 $31.2^{\circ}$ 的衍射峰则归属于 $\mathrm{PA}_{2}\left(\mathrm{CsPbr}_{3}\right) \mathrm{PbBr}_{4}$ 钻钛矿量子点(即 $\mathrm{PA}_{2}\left(\mathrm{CsPbr}_{3}\right)_{n-1}{ }^{-}$ $\left.\mathrm{PbBr}_{4}, n=2\right)$. XRD的结果, 不仅证实我们确实得到了
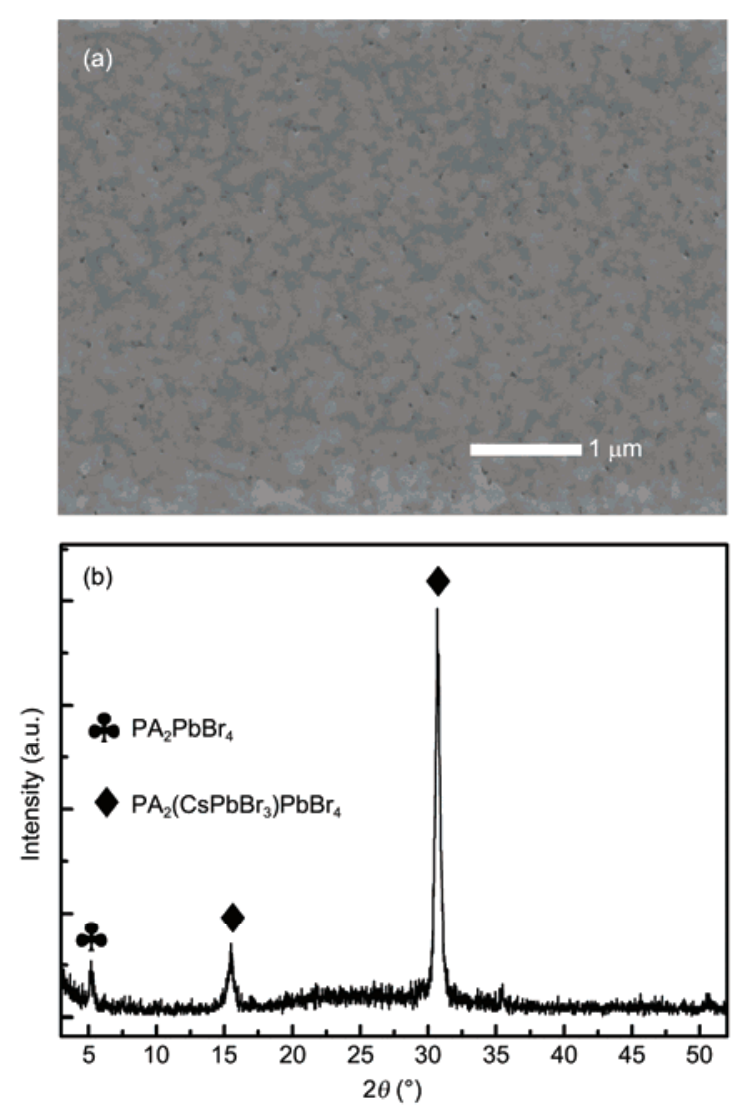

图 $2 \mathrm{PA}_{2}\left(\mathrm{CsPbBr}_{3}\right)_{n-1} \mathrm{PbBr}_{4}$ 二维层状钲钠矿薄膜的 $\mathrm{SEM}$ 表征(a)和 XRD表征(b)

Figure 2 SEM image (a) and XRD patterns (b) of $2 \mathrm{D} \mathrm{PA}_{2}\left(\mathrm{CsPbBr}_{3}\right)_{n-1^{-}}$ $\mathrm{PbBr}_{4}$ perovskites layer

$\mathrm{PA}_{2}\left(\mathrm{CsPbrr}_{3}\right)_{n-1} \mathrm{PbBr}_{4}$ 钙钛矿量子点, 也表明了 $\mathrm{PA}_{2}-$ $\left(\mathrm{CsPbBr}_{3}\right)_{n-1} \mathrm{PbBr}_{4}$ 钙钛矿量子点主要由 $\mathrm{PA}_{2} \mathrm{PbBr}_{4}$ $(n=1)$ 和 $\mathrm{PA}_{2}\left(\mathrm{CsPbr}_{3}\right) \mathrm{PbBr}_{4}(n=2)$ 这两种结构成分组 成. 除此之外, $\mathrm{PA}_{2}\left(\mathrm{CsPbBr}_{3}\right)_{n-1} \mathrm{PbBr}_{4}$ 钻钛矿量子点衍 射峰的半峰宽 $\left(\sim 0.6^{\circ}\right)$ 要明显大于 $\mathrm{CsPbr}_{3}$ 钙钛矿晶体 衍射峰的半峰宽 $\left(\sim 0.1^{\circ}\right)^{[17,18]}$, 说明PA的长链结构确 实了阻止 $\mathrm{CsPbBr}_{3}$ 钙钛矿单元的自组装生长, 使得晶 粒尺寸显著减小。

\section{$2.2 \mathrm{PA}_{2}\left(\mathrm{CsPbBr}_{3}\right)_{n-1} \mathrm{PbBr}_{4}$ 钻钛矿薄膜的光学 特性}

$\mathrm{PA}_{2}\left(\mathrm{CsPbBr}_{3}\right)_{n-1} \mathrm{PbBr}_{4}$ 钙钛矿薄膜的光学特性由 光致发光光谱(PL谱)和紫外吸收光谱(UVs谱)表征. 如图3(a)插图所示，在 365 $\mathrm{nm}$ 紫外灯的照射下， $\mathrm{PA}_{2}\left(\mathrm{CsPbBr}_{3}\right)_{n-1} \mathrm{PbBr}_{4}$ 钙钛矿薄膜发射出明亮的蓝绿 光. PL谱显示, 该发光峰位于 506 $\mathrm{nm}$, 半峰宽仅为 $\sim 18 \mathrm{~nm}$. 而对于 $\mathrm{CsPbBr}_{3}$ 钙钛矿晶体薄膜, 其PL发光 

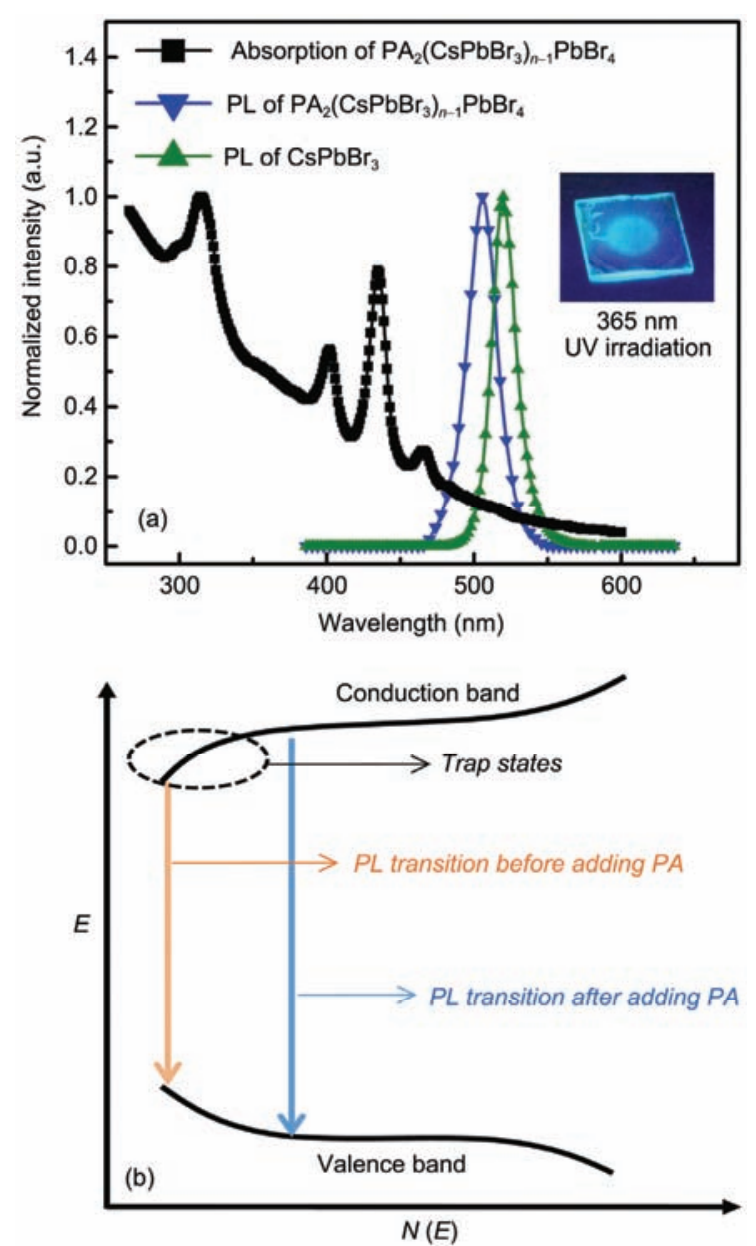

图 3 (网络版彩色) $\mathrm{PA}_{2}\left(\mathrm{CsPbBr}_{3}\right)_{n-1} \mathrm{PbBr}_{4}$ 钻钛矿薄膜的光学特性. (a) $\mathrm{PA}_{2}\left(\mathrm{CsPbr}_{3}\right)_{n-1} \mathrm{PbBr}_{4}$ 二维层状钙钛矿薄膜和 $\mathrm{CsPbr}_{3}$ 钻钛矿薄膜的 光致发光光谱(PL谱)和紫外吸收光谱(UVs谱); (b) $\mathrm{PA}_{2}\left(\mathrm{CsPbr}_{3}\right)_{n-1}$ $\mathrm{PbBr}_{4}$ 钙钛矿薄膜PL谱蓝移一种可能机制的示意图

Figure 3 (Color online) The optical properties of $\mathrm{PA}_{2}\left(\mathrm{CsPbr}_{3}\right)_{n-1}$ $\mathrm{PbBr}_{4}$ layer. (a) $\mathrm{PL}$ spectra of $\mathrm{PA}_{2}\left(\mathrm{CsPbBr}_{3}\right)_{n-1} \mathrm{PbBr}_{4}$ and $\mathrm{CsPbBr}_{3}$ layers, as well as UVs spectra of $\mathrm{PA}_{2}\left(\mathrm{CsPbBr}_{3}\right)_{n-1} \mathrm{PbBr}_{4}$ layer; (b) scheme of a possible mechanism for PL blue-shift of $\mathrm{PA}_{2}\left(\mathrm{CsPbBr}_{3}\right)_{n-1} \mathrm{PbBr}_{4}$ layer

峰位于 $522 \mathrm{~nm}$. 可见, $\mathrm{PABr}$ 的引人使 $\mathrm{CsPbBr}_{3}$ 的 $\mathrm{PL}$ 谱产生了明显的蓝移. 另外, $\mathrm{PA}_{2}\left(\mathrm{CsPbBr}_{3}\right)_{n-1} \mathrm{PbBr}_{4}$ 和 $\mathrm{CsPbr}_{3}$ 钙钛矿薄膜的苂光量子产率 (PLQY) 分别为 $3.8 \%$ 和 $2.7 \%$, 表明二维 $\mathrm{PA}_{2}\left(\mathrm{CsPbBr}_{3}\right)_{n-1} \mathrm{PbBr}_{4}$ 钙钛矿 的苂光量子产率更高.

图3(b) 展示了 $\mathrm{PA}_{2}\left(\mathrm{CsPbBr}_{3}\right)_{n-1} \mathrm{PbBr}_{4}$ 钻钛矿薄膜 的紫外吸收谱. 可以看到, $\mathrm{PA}_{2}\left(\mathrm{CsPbr}_{3}\right)_{n-1} \mathrm{PbBr}_{4}$ 钙钛 矿量子点在 466.5, 436.5, 402.7和 314.5 nm 4处产 生了明显的吸收峰, 而 $\mathrm{CsPbBr}_{3}$ 钙钛矿晶体仅在 $\sim 520$ $\mathrm{nm}$ 一处存在吸收峰 ${ }^{[18]}$. 紫外吸收谱的结果不仅证实 了 $\mathrm{PA}_{2}\left(\mathrm{CsPbBr}_{3}\right)_{n-1} \mathrm{PbBr}_{4}$ 钙钛矿量子点的带隙确实大
于 $\mathrm{CsPbBr}$ 钙钛矿晶体的带隙, 而且在光激发条件下, $\mathrm{PA}_{2}\left(\mathrm{CsPbr}_{3}\right)_{n-1} \mathrm{PbBr}_{4}$ 比 $\mathrm{Cs} \mathrm{PbBr}_{3}$ 产生了更丰富的激发 态 (因为 $\mathrm{PA}_{2}\left(\mathrm{CsPbBr}_{3}\right)_{n-1} \mathrm{PbBr}_{4}$ 有 4 处吸收峰, 而 $\mathrm{CsPbBr}_{3}$ 仅有 1 处吸收峰)。尽管 $\mathrm{PA}_{2}\left(\mathrm{CsPbr}_{3}\right)_{n-1} \mathrm{PbBr}_{4}$ 的激发态十分丰富，但是其PL最终却仅展现了单一 的发光峰. 对于该光学特性, 有以下两种可能的解 释: (1) $\mathrm{PA}_{2}\left(\mathrm{CsPbBr}_{3}\right)_{n-1} \mathrm{PbBr}_{4}$ 仅有一种激发态能以辐 射退激的方式进行发光, 而其他的激发态都以非辐 射退激的方式将能量耗散掉; (2) $\mathrm{PA}_{2}\left(\mathrm{CsPbBr}_{3}\right)_{n-1}$ $\mathrm{PbBr}_{4}$ 的激发态按能量由高到低, 依次将能量以 Föster能量转移的方式传递给能量最低的激发态, 最 后由能量最低的这个激发态进行辐射退激发光 ${ }^{[9]}$. 关 于 $\mathrm{PA}_{2}\left(\mathrm{CsPbBr}_{3}\right)_{n-1} \mathrm{PbBr}_{4}$ 的激发态特性比较复杂, 需 在将来的工作中进一步研究.

对于 $\mathrm{PA}_{2}\left(\mathrm{CsPbBr}_{3}\right)_{n-1} \mathrm{PbBr}_{4}$ 钙钛矿薄膜, 其 $\mathrm{PL}$ 谱 蓝移的一种可能机制, 如图3(b)所示. 在未加人 $\mathrm{PABr}$ 前, $\mathrm{CsPbBr}_{3}$ 自组装形成三维的钲钛矿晶体. 三维钙 钛矿晶体形成的薄膜往往存在大量陷阱态 (trap states), 这些陷阱态存在于三维钻钛矿晶体的内部 (bulk)或者三维钙钛矿晶体的边界(boundaries) ${ }^{[20]}$. 陷 阱态辅助的辐射退激过程 (trap-assisted recombination process)主要来自于电子在带边 (band edge)陷阱态能 级的辐射跃迁过程. 加人 $\mathrm{PABr}$ 后, 一方面在尺寸上, $\mathrm{CsPbBr} 3$ 三维钙钛矿晶体缩小成为 $\mathrm{PA}_{2}\left(\mathrm{CsPbBr}_{3}\right)_{n-1}-$ $\mathrm{PbBr}_{4}$ 钙钛矿量子点, 激子的束缚能增大, 这就抑制 了三维钙钛矿晶体的内部缺陷态的生成. 另一方面, $\mathrm{PA}_{2}\left(\mathrm{CsPbBr}_{3}\right)_{n-1} \mathrm{PbBr}_{4}$ 钙钛矿量子点能够形成连续、致 密的二维层状薄膜, 成膜质量大大提高, 这也抑制了 钙钛矿晶界处的缺陷态的生成. 总之, 加人 $\mathrm{PABr}$ 后, 使得 $\mathrm{PA}_{2}\left(\mathrm{CsPbBr}_{3}\right)_{n-1} \mathrm{PbBr}_{4}$ 二维层状钙钛矿薄膜中的 陷阱态大大减少, 这样原先的陷阱态辅助辐射退激 过程(带边陷阱态能级间的跃迁)转化成钙钛矿量子 点内部(带隙能级间的跃迁)的辐射退激过程. 由于电 子跃迁能隙的拓宽, 因此在 $\mathrm{PA}_{2}\left(\mathrm{CsPbBr}_{3}\right)_{n-1} \mathrm{PbBr}_{4}$ 二 维层状钙钛矿薄膜中, 光谱发生蓝移. 同时由于陷陎 态的减少, 激子的非辐射退激过程被抑制, 因此二维 层状钙钛矿薄膜的苂光效率也能得到增强 ${ }^{[3,4]}$.

\subsection{PeLED的电流-亮度-电压 $(I-L-V)$ 特性曲线}

以 $\mathrm{PA}_{2}\left(\mathrm{CsPbBr}_{3}\right)_{n-1} \mathrm{PbBr}_{4}$ 二维层状钙钛矿薄膜作 为发光层, 制备PeLED, 电流-亮度-电压 $(I-L-V)$ 特性 曲线如图4所示. 其中, 图4(a)展示了PeLED的电流 

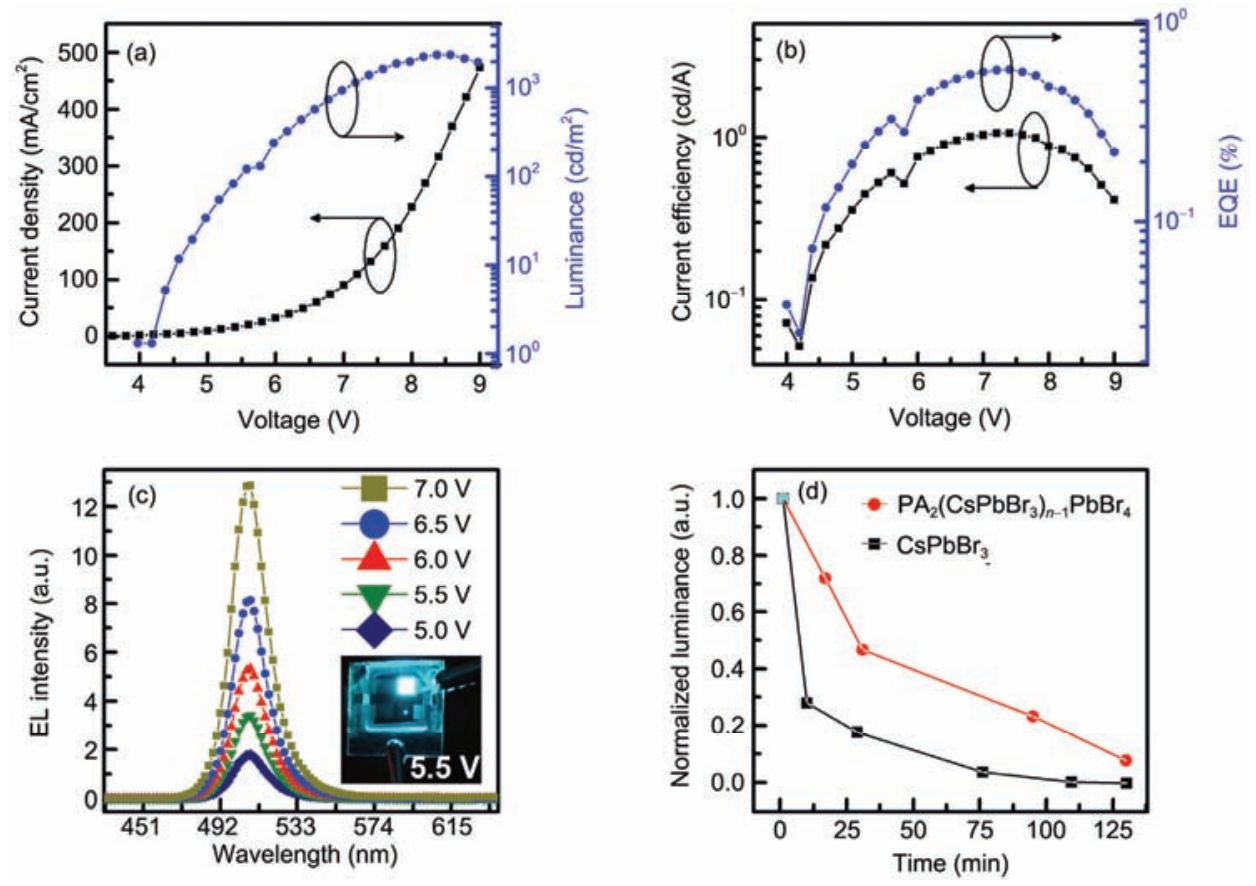

图 4 (网络版彩色)PeLED的器件结果. (a) 电流密度-电压 $(J-V)$ 曲线和亮度-电压 $(L-V)$ 曲线; (b) 电流效率-电压(current efficiency- $V$ )曲线和外量 子效率-电压(EQE- $V$ )曲线; (c) 不同偏压下的EL谱; (d) $\mathrm{PA}_{2}\left(\mathrm{CsPbr}_{3}\right)_{n-1} \mathrm{PbBr}_{4}$ 器件和 $\mathrm{CsPbBr}_{3}$ 器件的发光稳定性比较

Figure 4 (Color online) The EL performance of PeLED. (a) $J-V$ and $L-V$ characteristics; (b) current efficiency- $V$ and EQE- $V$ characteristics; and (c) EL spectra under different voltage bias of PeLED; (d) comparison of luminance stability of $\mathrm{PA}_{2}\left(\mathrm{CsPbBr}_{3}\right)_{n-1} \mathrm{PbBr}_{4} \mathrm{PeLED}_{\text {and }} \mathrm{CsPbBr} \mathrm{PeLED}_{3}$

密度-电压 $(J-V)$ 曲线和亮度-电压 $(L-V)$ 曲线. 与之前 报道的PeLED结果类似 ${ }^{[13,15]}, J-V$ 曲线和 $L-V$ 曲线都呈 现出典型的二极管特性, 即外加偏压超过器件的开 启电压 $(\sim 4.0 \mathrm{~V})$ 和启亮电压 $(\sim 4.2 \mathrm{~V})$ 后, 随着偏压的 增高, 器件的电流密度和亮度分别呈现出指数型的 增长. 值得注意的是, 该PeLED的电流密度 $\left(10^{-2} \sim 10^{2}\right.$ $\left.\mathrm{mA} / \mathrm{cm}^{2}\right)$ 较通常PeLED的电流密度 $\left(10^{-4} \sim 10^{4} \mathrm{~mA} / \mathrm{cm}^{2}\right)$ 要小很多 ${ }^{[15]}$. 这就说明, 均匀致密的 $\mathrm{PA}_{2}\left(\mathrm{CsPbBr}_{3}\right)_{n-1^{-}}$ $\mathrm{PbBr}_{4}$ 二维层状钙钛矿薄膜可以有效地阻挡器件中的 漏电流, 从而提升载流子的复合效率和激子的辐射 退激速率. $\mathrm{PA}_{2}\left(\mathrm{CsPBr}_{3}\right)_{n-1} \mathrm{PbBr}_{4}$ 器件的启亮电压为 $\sim 4.2 \mathrm{~V}$, 比之前报道的 $\mathrm{CsPbBr}_{3}$ 器件的启亮电压(通常 为 3.0 V $)^{[17,18]}$ 略高, 这是由于该PeLED要激发宽带 隙的 $\mathrm{PA}_{2}\left(\mathrm{CsPbBr}_{3}\right)_{n-1} \mathrm{PbBr}_{4}$ 所致. 当外加偏压达到 8.6 $\mathrm{V}$ 时, 器件的亮度达到最大, 最大亮度为 2370 $\mathrm{cd} / \mathrm{m}^{2}$. 之后, 再增加偏压, 大电流引起的焦耳热使 得器件开始老化衰减, 于是亮度逐渐下降. PeLED的 电流效率-电压(current efficiency- $V$ ) 曲线和外量子效 率-电压(EQE- $V$ )曲线如图4(b) 所示. 其中, PeLED的 外量子效率由文献[21]提供的方法计算得到. 可以看 到, 随着电压的升高, PeLED的电流效率和外量子效
率也逐渐升高. 当外加偏压达到7.4 V 时, 器件的电 流效率和外量子效率同时达到最大, 分别为 1.06 $\mathrm{cd} / \mathrm{A}$ 和 $0.57 \%$. 随后, PeLED在大偏压下出现老化, 器件的电流效率和外量子效率开始下降. 最后, 比较 了 $\mathrm{PA}_{2}\left(\mathrm{CsPbr}_{3}\right)_{n-1} \mathrm{PbBr}_{4}$ 器件和 $\mathrm{CsPbBr}_{3}$ 器件的发光稳 定性, 结果如图4(d)所示. 可以看到, $\mathrm{PA}_{2}\left(\mathrm{CsPbBr}_{3}\right)_{n-1}$ $\mathrm{PbBr}_{4}$ 器件在发光稳定性方面要优于 $\mathrm{CsPbr}_{3}$ 器件.

尽管目前绿光 $\mathrm{PeLED}\left(\right.$ 主要以 $\mathrm{CH}_{3} \mathrm{NH}_{3} \mathrm{PbBr}_{3}$ 或 $\mathrm{CsPbBr}_{3}$ 钙钛矿材料作为发光层) 已经取得了显著的 发展 ${ }^{[14,18,19]}$, 但是关于蓝光PeLED的文献报道却很 少. 传统得到蓝光PeLED的方法是将 $\mathrm{CH}_{3} \mathrm{NH}_{3} \mathrm{PbBr}_{3}$ 或 $\mathrm{CsPbBr}_{3}$ 钙钛矿中卤素离子进行部分替换, 即将 $\mathrm{Br}^{-}$取 代成 $\mathrm{Cl}^{-}$, 以此来调节钙钛矿的能级 ${ }^{[6]}$. 然而, 通过 该方法得到的 $\mathrm{CH}_{3} \mathrm{NH}_{3} \mathrm{PbCl}_{x} \mathrm{Br}_{3-x}$ 或 $\mathrm{CsPbCl}_{x} \mathrm{Br}_{3-x}$ 主要 存在以下 3 方面的问题: (1) $\mathrm{CH}_{3} \mathrm{NH}_{3} \mathrm{PbCl}_{x} \mathrm{Br}_{3-x}$ 或 $\mathrm{CsPbCl}_{x} \mathrm{Br}_{3-x}$ 的溶解性很差; (2) 难以通过一步旋涂的 方法得到高质量的 $\mathrm{CH}_{3} \mathrm{NH}_{3} \mathrm{PbCl}_{x} \mathrm{Br}_{3-x}$ 或 $\mathrm{CsPbl}_{x} \mathrm{Br}_{3-x}$ 钲钛矿薄膜; (3) $\mathrm{CH}_{3} \mathrm{NH}_{3} \mathrm{PbCl}_{x} \mathrm{Br}_{3-x}$ 或 $\mathrm{CsPbCl}_{x} \mathrm{Br}_{3-x}$ 的 光致发光效率和电致发光效率都很低 (通常 $<0.1$ $\mathrm{cd} / \mathrm{A})^{[6,12]}$. 因此, 通过溶液加工法得到的 $\mathrm{CH}_{3} \mathrm{NH}_{3}-$ $\mathrm{PbCl}_{x} \mathrm{Br}_{3-x}$ 或 $\mathrm{CsPbl}_{x} \mathrm{Br}_{3-x}$ 蓝光 $\mathrm{PeLED}$, 效果不理想. 
相比之下, 本文采用二维钙钛矿材料得到蓝光PeLED 的方法, 可以有效地解决上述 3 个问题. 但从表征结 果上看, 目前 $\mathrm{PA}_{2}\left(\mathrm{CsPbBr}_{3}\right)_{n-1} \mathrm{PbBr}_{4}$ 器件依旧处于蓝 绿光发射. 这是因为 $\mathrm{PA}_{2}\left(\mathrm{CsPbBr}_{3}\right)_{n-1} \mathrm{PbBr}_{4}$ 量子点中 的“量子阱”结构中存在能量转移过程, 发光仅由能 量最低的激子进行 (如图2(a)所示的单一发光峰). 所 以, $\mathrm{PA}_{2}\left(\mathrm{CsPbBr}_{3}\right)_{n-1} \mathrm{PbBr}_{4}$ 量子点蓝移也较小. 至于是 否会得到真正蓝光范畴的PeLED, 还需要未来进一 步的实验进行证实.

\section{3 结论}

本文采用 $\mathrm{PABr}$ 与 $\mathrm{CsPbBr}_{3}$ 前驱体溶液共混的策 略, 让PA离子取代 $\mathrm{CsPbBr}_{3}$ 钙钛矿中的部分 $\mathrm{Cs}$ 离子, 利用 $\mathrm{PABr}$ 的长链结构对 $\mathrm{CsPbBr}_{3}$ 钙钛矿单元的自组 装生长进行有效阻止, 合成了纳米级的 $\mathrm{PA}_{2}-$ $\left(\mathrm{CsPbBr}_{3}\right)_{n-1} \mathrm{PbBr}_{4}$ 钙钛矿量子点. 该 $\mathrm{PA}_{2}\left(\mathrm{CsPbBr}_{3}\right)_{n-1}{ }^{-}$
$\mathrm{PbBr}_{4}$ 钙钛矿量子点主要由 $\mathrm{PA}_{2} \mathrm{PbBr}_{4}$ 和 $\mathrm{PA}_{2}\left(\mathrm{CsPbr}_{3}\right)$ $\mathrm{PbBr}_{4}$ 两种钙钛矿结构组成. 形成的二维层状钻钛矿 薄膜均匀致密, 可以有效地阻挡器件中的漏电流. 在 光致发光条件下, $\mathrm{PA}_{2}\left(\mathrm{CsPbBr}_{3}\right)_{n-1} \mathrm{PbBr}_{4}$ 二维层状钙 钛矿薄膜呈现明亮的蓝绿光发射(发光峰位于 506 $\mathrm{nm})$, 较 $\mathrm{CsPbBr}_{3}$ 三维钙钛矿晶体薄膜的绿光发射 $(\sim 522 \mathrm{~nm})$ 出现了明显的蓝移. 在电致发光方面, 基 于 $\mathrm{PA}_{2}\left(\mathrm{CsPbBr}_{3}\right)_{n-1} \mathrm{PbBr}_{4}$ 的 $\mathrm{PeLED}$ 同样为蓝绿光发射 $(\sim 508 \mathrm{~nm})$, 启亮电压为 $\sim 4.2 \mathrm{~V}$, 最大亮度为 2370 $\mathrm{cd} / \mathrm{m}^{2}$, 最高电流效率为 $\sim 1.06 \mathrm{~cd} / \mathrm{A}$, 最高 $\mathrm{EQE}$ 为 $\sim 0.57 \%$. 而且, 电致发光光谱表明, 不同偏压下, PeLEDs的发光特性没有发生改变. 最后, 相比于传 统的通过取代卤素离子得到蓝光PeLED的办法, 本 工作在制作工艺、成膜质量以及PeLED的发光效率都 得到了显著的提升, 为将来进一步探索低成本、高效 率的蓝光PeLEDs提供了一种可行的思路.

\section{参考文献}

1 Li X, Wu Y, Zhang $\mathrm{S}$, et al. $\mathrm{CsPbX}_{3}$ quantum dots for lighting and displays: Room-temperature synthesis, photoluminescence superiorities, underlying origins and white light-emitting diodes. Adv Funct Mater, 2016, 26: 2435-2445

2 Kim Y, Cho H, Heo J H, et al. Multicolored organic/inorganic hybrid perovskite light-emitting diodes. Adv Mater, 2015, 27: 1248-1254

3 Xiao Z, Kerner R A, Zhao L, et al. Efficient perovskite light-emitting diodes featuring nanometre-sized crystallites. Nat Photonics, 2017, 11: $108-116$

4 Zhao L, Yeh Y, Tran N L, et al. In situ preparation of metal halide perovskite nanocrystal thin films for improved light-emitting devices. ACS Nano, 2017, 11: 3957-3964

5 Hong W, Huang Y, Chang C. Efficient low-temperature solution-processed lead-free perovskite infrared light-emitting diodes. Adv Mater, 2016, 28: 8029-8036

6 Sadhanala A, Ahmad S, Zhao B, et al. Blue-green color tunable solution processable organolead chloride-bromide mixed halide perovskites for optoelectronic applications. Nano Lett, 2015, 15: 6095-6101

7 Cho H, Jeong S, Park M, et al. Overcoming the electroluminescence efficiency limitations of perovskite light-emitting diodes. Science, 2015, 350: 1222-1225

8 Deng W, Xu X, Zhang X, et al. Organometal halide perovskite quantum dot light-emitting diodes. Adv Funct Mater, 2016, 26, 4797-4802

9 Wang N, Cheng L, Ge R, et al. Perovskite light-emitting diodes based on solution-processed self-organized multiple quantum wells. Nat Photonics, 2016, 10: 699-704

10 Zhao Y, Zhu K. Organic-inorganic hybrid lead halide perovskites for optoelectronic and electronic applications. Chem Soc Rev, 2016, 45: 655-689

11 Li G, Price M, Deschler F. Research update: Challenges for high-efficiency hybrid lead-halide perovskite LEDs and the path towards electrically pumped lasing. APL Mater, 2016, 4: 091507

12 Tan Z K, Moghaddam R S, Lai M L, et al. Bright light-emitting diodes based on organometal halide perovskite. Nat Nanotech, 2014, 9: 687-692

13 Chen P, Xiong Z, Wu X, et al. Highly efficient perovskite light-emitting diodes incorporating full film coverage and bipolar charge injection. J Phys Chem Lett, 2017, 8: 1810-1818

14 Zhang X, Wang W, Xu B, et al. Thin film perovskite light-emitting diode based on $\mathrm{CsPbr}_{3}$ powders and interfacial engineering. Nano Energy, 2017, 37: 40-45

15 Xiong Z Y, Gao C H, Wang Z Q, et al. White PeLED employing a mixed emission layer composed of a small organic molecule and an 
organicinorganic hybrid perovskite (in Chinese). Chin Sci Bull, 2017, 62: 2780-2787 [熊自阳，高春红，王治强，等. 基于有机小分子 和有机-无机杂化钲钛矿复合发光层的白光 PeLED. 科学通报, 2017, 62: 2780-2787

16 Tsai H, Nie W, Blancon J C, et al. High-efficiency two-dimensional ruddlesden-popper perovskite solar cells. Nature, 2016, 536: 312-316

17 Ling Y, Tian Y, Wang X, et al. Enhanced optical and electrical properties of polymer-assisted all-inorganic perovskites for light-emitting diodes. Adv Mater, 2016, 28: 8983-8989

18 Li J, Shan X, Bade S G R, et al. Single-layer halide perovskite light-emitting diodes with sub-band gap turn-on voltage and high brightness. J Phys Chem Lett, 2016, 7: 4059-4066

19 Weidman M C, Seitz M, Stranks S D, et al. Highly tunable colloidal perovskite nanoplatelets through variable cation, metal, and halide composition. ACS Nano, 2016, 10: 7830-7839

20 Shao $\mathrm{Y}$, Xiao Z, Bi C, et al. Origin and elimination of photocurrent hysteresis by fullerene passivation in $\mathrm{CH}_{3} \mathrm{NH}_{3} \mathrm{PbI}_{3} \mathrm{planar}$ heterojunction solar cells. Nat Commun, 2014, 5: 5784

21 Kawamura Y, Sasabe H, Adachi C. Simple accurate system for measuring absolute photoluminescence quantum efficiency in organic solid-state thin films. Jpn J Appl Phys, 2004, 43: 7729-7730 
Summary for “基于 $\mathrm{PA}_{2}\left(\mathrm{CsPBr}_{3}\right)_{n-1} \mathrm{PbBr}_{4}$ 二维层状钻钛矿的电致发光二极管”

\title{
Light-emitting diodes based on two-dimensional $\mathrm{PA}_{2}\left(\mathrm{CsPbBr}_{3}\right)_{n-1} \mathrm{PbBr}_{4}$ layered perovskites
}

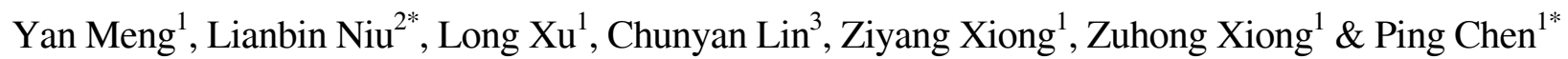 \\ ${ }^{1}$ MOE Key Laboratory on Luminescence and Real-Time Analysis, School of Physical Science and Technology, Southwest University, Chongqing \\ 400715, China; \\ ${ }^{2}$ Chongqing Key Laboratory of Optical Electronic Functional Materials, College of Physics and Electronic Engineering, Chongqing Normal University, \\ Chongqing 401331, China; \\ ${ }^{3}$ Chongqing Key Laboratory for Advanced Materials and Technologies of Clean Energy, Institute for Clean Energy and Advanced Materials \\ (ICEAM), Southwest University, Chongqing 400715, China \\ *Corresponding authors, E-mail: niulb03@126.com; chenping206@126.com
}

Solution-processed organometal halide perovskites (formulated as $\mathrm{ABX}$, where $\mathrm{A}$ is the methylammonium $\left(\mathrm{CH}_{3} \mathrm{NH}_{3}^{+}\right.$) (MA) or metal cesium cation $\left(\mathrm{Cs}^{+}\right)$, $\mathrm{B}$ is the lead cation $\left(\mathrm{Pb}^{2+}\right)$ and $\mathrm{X}$ is the halide anion $\left(\mathrm{Br}^{-}, \mathrm{I}^{-}, \mathrm{Cl}^{-}\right)$) are promising candidates for next generation light-emitting materials owing to their unique optoelectronic properties. These properties mainly include extremely high photoluminescence quantum yield (PLQY), easily tunable band gap and narrow emission characteristics. During the past two years, impressive progresses have been made in perovskite light-emitting diodes (PeLED) with hybrid organic-inorganic perovskite materials (i.e., $\mathrm{CH}_{3} \mathrm{NH}_{3} \mathrm{PbBr}_{3}$ ). So far, the best external quantum efficiency (EQE) of $\mathrm{CH}_{3} \mathrm{NH}_{3} \mathrm{PbBr}_{3}$-based PeLED was reaching up to $~ 8.53 \%$ which was close to the results of organic light-emitting diodes (OLED). Despite the remarkable performance of the devices demonstrated, the stability of organic-inorganic hybrid perovskites remains a major concern. To circumvent this problem, all-inorganic halide perovskites exhibiting higher thermal and chemical stability, such as cesium lead bromide $\left(\mathrm{CsPbr}_{3}\right)$, have been used as alternative emitters in PeLED. Despite the advantages described above, both the brightness and the EQE of $\mathrm{CsPBBr}_{3}$-based PeLED remain limited. One important reason for low efficiency of $\mathrm{CsPbr}_{3}$-based $\mathrm{PeLED}$ is the facile dissociation of excitons in 3D perovskites due to their small exciton binding energy. One promising way to enhance the exciton binding energy is reducing the grain size of perovskites. Herein, we report a solution process to form highly uniform and ultra-flat perovskite films with nanometre-sized grains that allow us to demonstrate efficient PeLED. By mixing long-chain ammonium halides of $\mathrm{CH}_{3} \mathrm{CH}_{2} \mathrm{CH}_{2} \mathrm{NH}_{3} \mathrm{Br}(\mathrm{PABr})$ with the $3 \mathrm{D} \mathrm{CsPbBr}_{3}$ perovskite precursor solution, the growth of 3D perovskite grains are dramatically impeded and the $\mathrm{PA}_{2}\left(\mathrm{CsPBrr}_{3}\right)_{n-1} \mathrm{PbBr}_{4}$ quantum dots with grain size of $\sim 100 \mathrm{~nm}$ were achieved. This is because the long-chain ammonium ions cannot fill the corner of $\mathrm{PbBr}_{4}$ octahedral layers and therefore impedes the 3D perovskite formation. Moreover, the nanometre-sized grains feature reduced dimensionality, starting a transition from 3D to 2D layered (so-called Ruddlesden-Popper phase) perovskite structures. The SEM image show the morphology of $\mathrm{PA}_{2}\left(\mathrm{CsPbBr}_{3}\right)_{n-1} \mathrm{PbBr}_{4}$ layer was dense and uniform, which is very important to suppress the leaking current in the PeLED. We found that $\mathrm{PA}_{2}\left(\mathrm{CsPbBr}_{3}\right)_{n-1} \mathrm{PbBr}_{4}$ film mainly consists of two different 2D structures, namely $\mathrm{PA}_{2} \mathrm{PbBr}_{4}(n=1)$ and $\mathrm{PA}_{2}\left(\mathrm{CsPbBr}_{3}\right) \mathrm{PbBr}_{4}(n=2)$, according to the analysis of XRD pattern. Compared to the single UVs absorption peak of $\mathrm{CsPbBr}_{3}$ at $\sim 520 \mathrm{~nm}, \mathrm{PA}_{2}\left(\mathrm{CsPbBr}_{3}\right)_{n-1} \mathrm{PbBr}_{4}$ film show multiple absorption peaks, indicating several extonic states are formed upon light excitation. Although several extonic states exist, photoluminescence (PL) with only one peak of $506 \mathrm{~nm}$ was observed in $\mathrm{PA}_{2}\left(\mathrm{CsPbBr}_{3}\right)_{n-1} \mathrm{PbBr}_{4}$ film. We suspect that the energy transfer occurs from the excitons with larger bandgap to those with smaller bandgap, making recombination occurs only in the excitons with the smallest bandgap. For the PeLED with structure of ITO/PEDOT: $\mathrm{PSS} / \mathrm{PA}_{2^{-}}$ $\left(\mathrm{CsPbBr}_{3}\right)_{n-1} \mathrm{PbBr}_{4} / \mathrm{TPBi} / \mathrm{Cs}_{2} \mathrm{CO}_{3} / \mathrm{Al}$, the turn-on voltage is $\sim 4.2 \mathrm{~V}$, the maximum luminance is $\sim 2370 \mathrm{~cd} / \mathrm{m}^{2}$, the maximum current efficiency is $\sim 1.06 \mathrm{~cd} / \mathrm{A}$, and maximum $\mathrm{EQE}$ is $\sim 0.57 \%$. Compared to the traditional method achieving blue PeLED by substituting halide anions, obvious improvements can be seem not only in the material processing and the perovskites film quality, but also in the PeLED's performance. Our studies may provide an alternative way to explore low-cost and high- efficiency blue PeLED.

\section{$\mathrm{PA}_{2}\left(\mathrm{CsPbBr}_{3}\right)_{n-1} \mathrm{PbBr}_{4}$, two-dimensional layer, perovskite, PeLED}

doi: 10.1360/N972017-00712 